\title{
Hermetic Seal in Obturation: An Achievable Goal with Recently Introduced Cpoint
}

\author{
Rani Somani ${ }^{1}$, Shipra Jaidka², Deepti J Singh ${ }^{3}$, Navpreet Kaur ${ }^{4}$
}

\begin{abstract}
Aim and objectives: To evaluate and compare the dentinal microcrack formation after obturation using CPoint and gutta percha as root canal filling materials.

Materials and methods: Forty orthodontically extracted single-rooted premolars were selected. The teeth were decoronated and were then divided into four groups $(n=10)$. In group I, samples were left unprepared and unfilled (negative control), in group II, samples were prepared and left unobturated (positive control), in group III, samples were prepared and obturation was done with Cpoint (F3) and endosequence bioceramic sealer, and in group IV, samples were prepared and obturation was done with Protaper gutta percha (F3) and endosequence bioceramic sealer. Then, the samples were kept for 1 week at $37^{\circ} \mathrm{C}$ and $100 \%$ humidity. All roots were then sectioned at $2 \mathrm{~mm}$ from the apex. Additional cross sections at a $4 \mathrm{~mm}$ level were made in groups III and IV. Then, the sections were observed under a scanning electron microscope and the presence of dentinal microcracks was checked.

Results: There was statistically nonsignificant difference for a mean number of microcracks in the samples after obturation with CPoint and gutta percha.

Conclusion: CPoint can be used as an alternative to gutta percha as it provides better seal because of its lateral hygroscopic expansion without causing significant damage to the dentinal microstructure.

Clinical significance: In spite of gutta percha being the gold standard in obturating material, still the desired hermetic seal could not be achieved. To achieve this attainable goal, a new material CPoint had been introduced, which has the ability to undergo hygroscopic expansion within the root canal.
\end{abstract}

Keywords: CPoint, Dentinal microcracks, Gutta-percha, Obturation.

International Journal of Clinical Pediatric Dentistry (2019): 10.5005/jp-journals-10005-1619

\section{INTRODUCTION}

Endo is a Greek word for "Inside" and Odont is a Greek word for "Tooth." Endodontic therapy treats the inside of the tooth and its success is based on the triad of thorough canal debridement, effective disinfection, and obturation of the canal space. Historically, a significant share of this triad has been allocated to obturation of the canal space. The ultimate goal of obturation of the root canal system is to prevent microorganisms from reentering the root canal and to isolate any microorganisms from the nutrients of tissue fluids which might percolate either from the apical area or the coronal region. ${ }^{1}$ To achieve this, an obturating material is needed to provide a hermetic seal in the root canal.

Since the introduction in 1867 by Bowman, gutta percha had been the standard obturation material utilized in root canal therapy. Even though gutta percha possesses many benign properties, including chemical stability, biocompatibility, nonporosity, radiopacity, and the ability to be manipulated and abstracted, it does not chemically bond to internal tooth structures. Moreover, due to the hydrophobic nature of the gutta percha, sealers tend to pull away from gutta percha on setting, resulting in incomplete seal and failure of obturation later on. ${ }^{2}$

To overcome these problems, the most recent advancement in endodontic obturating materials is to utilize a hydrophilic polymer in the root canal. The system consists of premade obturation points (CPoint) containing a polyamide core with an outer bonded hydrophilic polymer coating accompanied by a bioceramic sealer. ${ }^{3}$

These endodontic points are designed to expand laterally without expanding axially by absorbing residual moisture from the
${ }^{1-4}$ Department of Pedodontics and Preventive Dentistry, Divya Jyoti (DJ) College of Dental Sciences and Research, Modinagar, Uttar Pradesh, India

Corresponding Author: Rani Somani, Department of Pedodontics and Preventive Dentistry, Divya Jyoti (DJ) College of Dental Sciences and Research, Modinagar, Uttar Pradesh, India, Phone: +91 9810950754, e-mail: somanirani@gmail.com

How to cite this article: Somani R, Jaidka S, Singh DJ, et al. Hermetic Seal in Obturation: An Achievable Goal with Recently Introduced Cpoint. Int J Clin Pediatr Dent 2019;12(5):410-413.

Source of support: Nil

Conflict of interest: None

instrumented root canal space and naturally present moisture in the dentinal tubules. ${ }^{3}$ To date very few literature has been reported on this novel obturating material.

In a study, CPoint was found to be better as compared to gutta percha in terms of the adaptation of sealers and their penetration into simulated lateral canals (Arora and Hedge). ${ }^{4}$ Additionally, the fracture resistance of endodontically treated teeth was found to be higher when obturation was done with hydrophilic systems as compared to hydrophobic systems (Hegde and Arora). ${ }^{3}$ Furthermore, CPoint with a bioceramic sealer was found to be the best in providing coronal seal against bacterial leakage as compared to other obturating materials (Mobarak et al.). ${ }^{5}$

But as it had already been stated that endodontically treated teeth are more susceptible to fracture than the teeth with intact pulps, it became an imperative query whether the expansion of 
hydrophilic material CPoint might not weaken the unyielding dentinal canal space by causing an increase in stress, thus producing microcracks.

Therefore, the aim of the present study was to evaluate and compare the dentinal microcrack formation in endodontically treated teeth after obturation with different root canal filling materials.

\section{Materials and Methods}

The present in vitro study was carried out in the Department of Pedodontics and Preventive Dentistry, Divya Jyoti (DJ) College of Dental Sciences and Research, Modinagar, in collaboration with the Department of Physics, CCS University, Meerut.

Forty orthodontically extracted single rooted non-carious premolars with intact buccal or lingual surfaces were selected for the study. The selected teeth were autoclaved and cleaned using an ultrasonic scaler to remove the debris.

\section{Decoronation of Samples}

The selected teeth were decoronated in order to standardize the root length to $12 \mathrm{~mm}$. All the decoronated roots were stored in distilled water throughout the study in order to avoid dehydration.

\section{Division of Samples}

After decoronation, the roots were divided into four groups: in group I, neither biomechanical preparation nor obturation was done (negative control), whereas in group II, biomechanical preparation was done but no obturation was performed (positive control). In group III (experimental group), biomechanical preparation along with obturation with Cpoint and an endosequence bioceramic sealer was done. In group IV (experimental group), biomechanical preparation along with obturation with Protaper gutta percha and endosequence bioceramic sealer was done.

\section{Methodology}

\section{Biomechanical Preparation}

Biomechanical preparation was done in all the groups except in group I (unprepared and unfilled). As decoronation was already performed, no access cavity was prepared. The canal patency was achieved using a standard no. $10 \mathrm{~K}$ file and the pulp was extirpated with no. 10 broach. The working length was estimated by placing a $10 \mathrm{~K}$ size file into the root canal until it was observed at the apex. Then, with the help of radiograph, the working length at $12 \mathrm{~mm}$ was reconfirmed.

The root canals were biomechanically prepared using a $\mathrm{H}$ and Protaper file system up to size F3 (Dentsply Maillefer SA, Baillaigues, Switzerland). Irrigation was done with $1 \mathrm{~mL}$ of $5.25 \%$ sodium hypochlorite throughout the biomechanical preparation and finally with $3 \mathrm{~mL}$ of $17 \%$ EDTA for 1 minute once the biomechanical preparation was done according to manufacturer's instructions. Then, the canals were finally rinsed with distilled water.

\section{Obturation}

Obturation was done only in groups III and IV. For obturation, the canals were dried first, and then the endosequence $B C$ sealer (Brasseler USA, Savannah, GA) was introduced into the root canal via its intracanal tip. As the biomechanical preparation was done up to file size F3 of the hand Protaper file system, the obturation was done using a single cone technique with an $\mathrm{F} 3$ cone of CPoint (EndoTechnologies, LLC, Shrewsbury, MA, USA) in group III and an
F3 cone of Protaper gutta percha in group IV. Before obturation markings were made on an F3 master cone in both the groups at $2 \mathrm{~mm}$ and $12 \mathrm{~mm}$ from the apical tip of the cone, the apical $2 \mathrm{~mm}$ of the F3 master cone was cut with sterile stainless steel scissors and the remaining point was coated with a sealer and slowly inserted into the canal till a $2 \mathrm{~mm}$ short working length was achieved. The apical $2 \mathrm{~mm}$ decoronated roots were not obturated purposefully in groups III and IV.

Then, the samples were kept at $37^{\circ} \mathrm{C}$ and $100 \%$ humidity for the setting of the sealer.

\section{Microcrack Evaluation \\ Sectioning of Decoronated Roots}

All the roots of groups I to IV were crosssectioned at the level of $2 \mathrm{~mm}$ from the apex. But, in groups III and IV where obturation was done using CPoint and gutta- percha, respectively, additional cross sections were made at the $4 \mathrm{~mm}$ level from the apex. Sectioning was done using a slow speed diamond disc along with water as a coolant for each sample. A new disk was used for each sample.

\section{Microscopic Examination}

The samples were mounted on stubs and subjected to platinum sputtering. The samples were then transferred to a scanning electron microscope for image analysis. Thereafter, the images were evaluated to detect the presence of microcracks.

\section{SCORING For Microcracks}

For statistical purpose, the microcracks were given a score: absence of crack: 1, presence of one crack: 2, presence of two cracks : $2+$ $1=3$, presence of three cracks : $3+1=4$, and so on (Figs 1 to 3 ). The mean values for the number of microcracks were calculated accordingly. Data were collected, tabulated, and sent for statistical analysis.

\section{Results}

The data were subjected to statistical analysis using Statistical Package for the Social Sciences (SPSS) version 19. The $p$ value $<0.05$ is considered to be statistically significant and the one-way analysis of variance (ANOVA) test and an independent $t$ test were applied to compare the various groups.

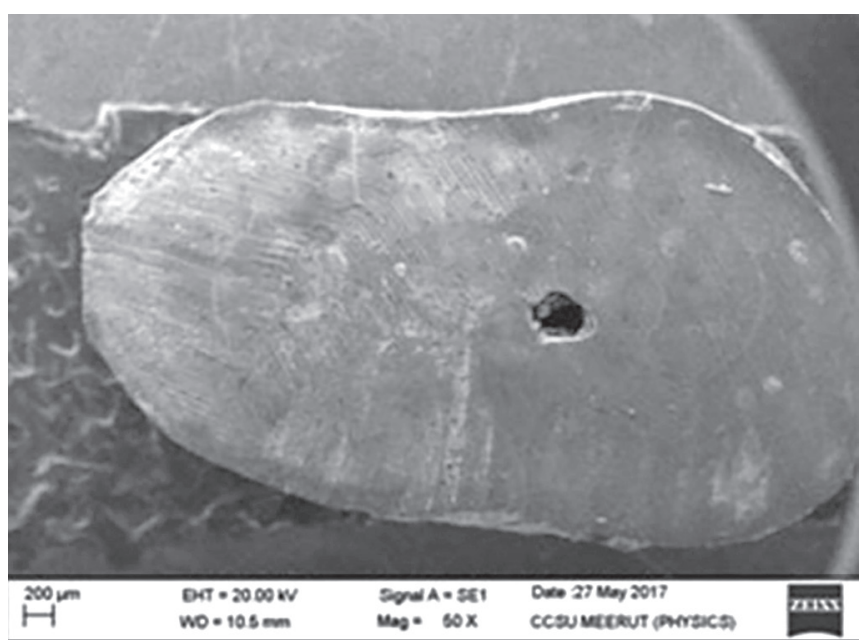

Fig. 1: Score 1: Absence of microcracks 


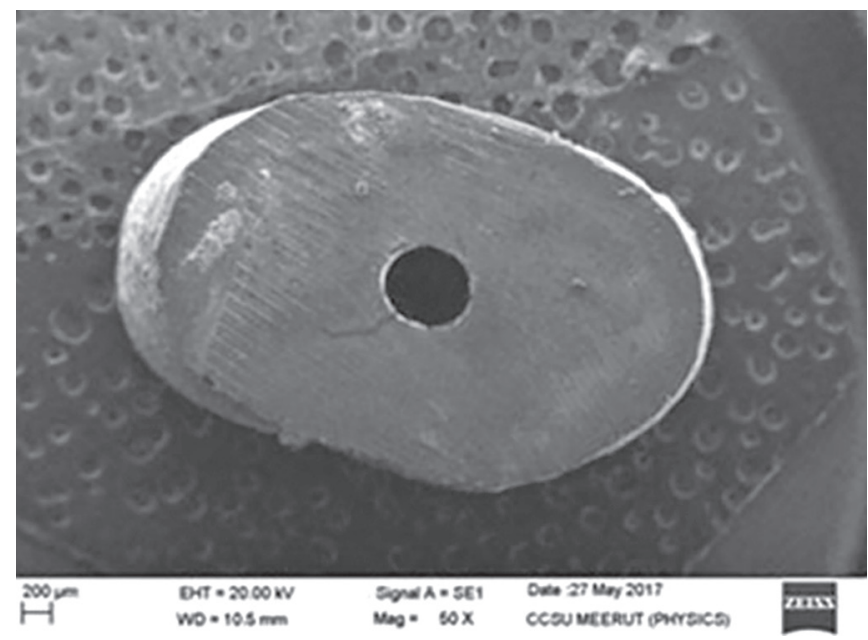

Fig. 2: Score 2: Presence of one microcrack

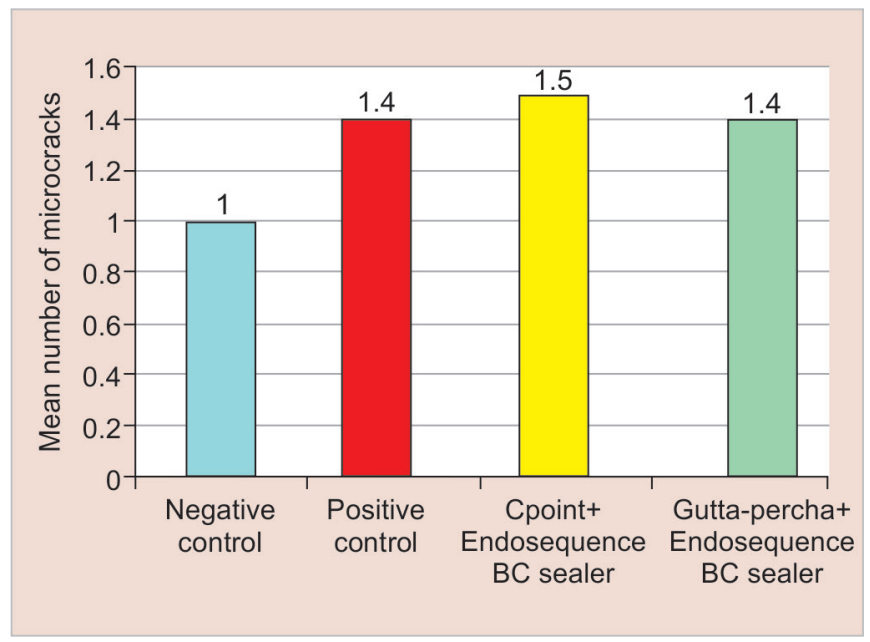

Fig. 4: Mean number of microcracks after biomechanical preparation

The mean number of microcracks formed after biomechanical preparation was found to be $1.00 \pm 0.00$ for group l, $1.40 \pm 0.51$ for group II, $1.50 \pm 0.77$ for group III, and $1.40 \pm 0.51$ for group IV. It was noted that comparable microcracks were seen in all the groups after biomechanical preparation (Table 1 and Fig. 4).

The mean number of microcracks formed after obturation in groups III and IV, where obturation was done with CPoint and gutta-percha, respectively, was found to be $1.70 \pm 0.823$ for group III and $1.40 \pm 0.51$ for group IV. When intercomparison between groups III and IV was done, a nonsignificant difference was found with a $p$ value of 0.342 (Table 2 and Fig. 5).

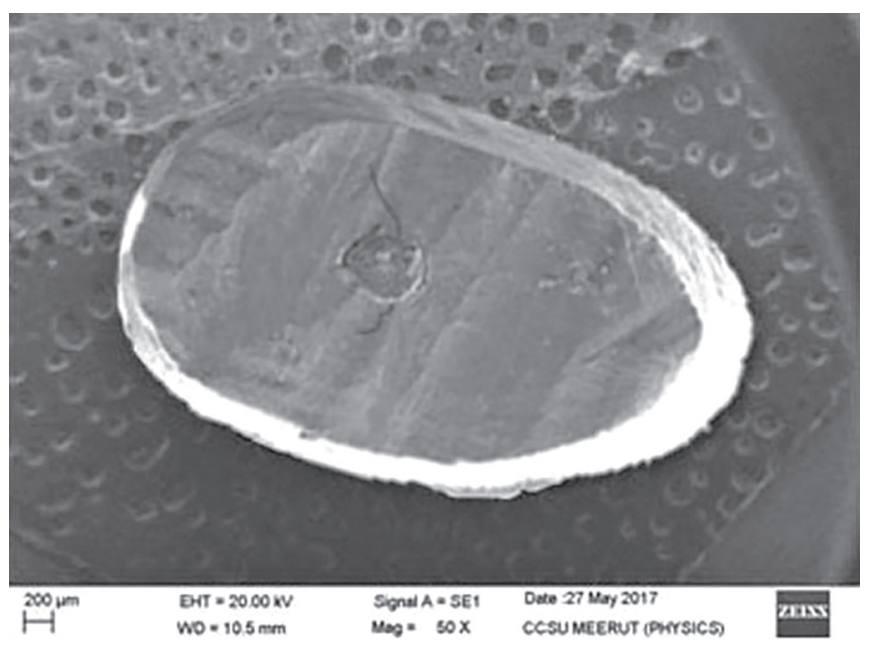

Fig. 3: Score 3: Presence of two microcracks

\section{Discussion}

It had already been reported that microcracks occur in dentin in varying numbers due to biomechanical preparation (Ashwinkumar et al., Kumaran et al.). ${ }^{6,7}$ But, the aim of our study was to evaluate the microcracks caused by obturating materials. Thus, to avoid the counting of the microcracks that might have been caused by biomechanical preparation in the counting of microcracks caused by obturating material, we had left a $2 \mathrm{~mm}$ apical region of decoronated roots without obturation in groups III and IV and sectioned the roots at two different levels, i.e., $2 \mathrm{~mm}$ (nonobturated part) and $4 \mathrm{~mm}$ (obturated part) from the apex, so that, we count only those additional cracks at $4 \mathrm{~mm}$ level sections which were not present at $2 \mathrm{~mm}$ level sections and thus were considered to be caused by the obturating material.

On statistical evaluation, the mean number of microcracks after obturation was found to be more in group III (obturation was done with CPoint) and less in group IV (obturation was done with gutta percha). But, when intercomparison was done between groups III and IV, it was found that the increase in the number of microcracks after obturation with CPoint was found to be nonsignificant when compared with the samples obturated with gutta percha.

CPoint is an obturation point consisting of an inner radiopaque nylon core of trogamid $T$ and trogamid $C X$ and an outer radiopaque hydrophilic polymer coating of acrylonitrile and vinylpyrrolidone. Because of the hydrophilic polymer coating of CPoint, it has the ability to absorb moisture from dentinal tubules and shows lateral hygroscopic expansion. ${ }^{8}$ According to manufacturers, the lateral expansion occurs non-isotropically meaning that it expands only till the time it comes in contact with the hard non-yielding dentinal

Table 1: Comparison of a number of microcracks after biomechanical preparation using the ANOVA test

\begin{tabular}{llllll}
\hline Groups & $N$ & Mean & Standard deviation & Fvalue & $p$ value \\
\hline Negative control & 10 & 1.00 & 0.000 & 1.903 & 0.147 (nonsignificant) \\
Positive control & 10 & 1.40 & 0.516 & & \\
CPoint + endosequence BC sealer & 10 & 1.50 & 0.707 & & \\
Gutta percha + endosequence BC sealer & 10 & 1.40 & 0.516 & \\
\hline
\end{tabular}

$p \leq 0.05$ significant using one-way ANOVA 
Hermetic Seal in Obturation: An Achievable Goal with Recently Introduced Cpoint

Table 2: Comparison of microcracks after obturation between groups III and IV using the independent $t$ test

\begin{tabular}{llllll}
\hline$G P$ & $N$ & Mean & Standard deviation & $t$ value & $p$ value \\
\hline CPoint + endosequence BC sealer & 10 & 1.70 & 0.823 & 0.976 & 0.342 (nonsignificant) \\
Gutta-percha + endosequence BC sealer & 10 & 1.40 & 0.516 & & \\
\hline$P \leq 0.05$
\end{tabular}

$p \leq 0.05$ significant using the independent $t$ test

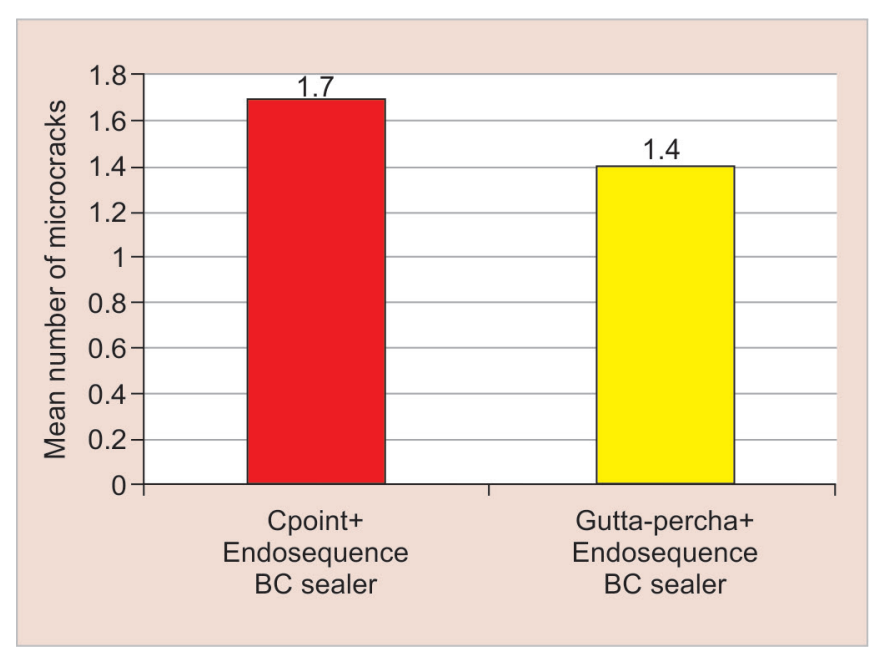

Fig. 5: Mean number of microcracks after obturation

wall. ${ }^{9}$ The increase in the number of microcracks after obturation with CPoint might be because of its lateral hygroscopic expansion.

Gutta percha is the transisomer of polyisoprene and consists primarily of zinc oxide (50-79\%), actual gutta percha (19-22\%), metal salts $(1-7 \%)$, and wax or resin $(1-4 \%){ }^{10}$ It is hydrophobic in nature and lacks the ability to expand laterally due to which the number of microcracks did not increase after obtuartion with gutta percha.

\section{Conclusion}

Within the limitation of this in vitro study, it was concluded that the mean number of microcracks was found to be more in the samples obturated with CPoint and less in the samples obturated with gutta percha, but the mean number of microcracks after obturation with CPoint was found to be comparable when compared with the samples obturated with gutta percha.

So, based on the above findings, we can recommend CPoint as an obturating material that provides better seal because of its lateral hygroscopic expansion without causing significant damage to the dentinal microstructure, but further studies with a larger sample size are required to authenticate the results.

\section{Clinical Significance}

In spite of gutta percha being the gold standard in obturating material, still the desired hermetic seal could not be achieved. To achieve this attainable goal, a new material CPoint had been introduced which has the ability to undergo hygroscopic expansion within the root canal.

\section{References}

1. Li G, Niu L, et al. Ability of new obturation materials to improve the seal of the root canal system-a review. Acta Biomater 2014;10(3): 1050-1063. DOI: 10.1016/j.actbio.2013.11.015.

2. Hegde V, Arora S. Sealing ability of a novel hydrophilic vs conventional hydrophobic obturation systems: a bacterial leakage study. J Conserv Dent 2015;18(1):62-65. DOI: 10.4103/0972-0707.148898.

3. Hegde V, Arora S. Fracture resistance of roots obturated with novel hydrophilic obturation systems. J Conserv Dent 2015;18(3):261-264. DOI: 10.4103/0972-0707.154047.

4. Arora S, Hegde V. Comparative evaluation of a novel smart-seal obturating system and its homogeneity of using cone beam computed tomography: in vitro simulated lateral canal study. J Conserv Dent 2014;17(4):364-368. DOI: 10.4103/0972-0707.136512.

5. Mobarak A, Moussa S, et al. Comparison of bacterial coronal leakage between different obturation materials (an in vitro study). Alex Dent J 2015;40:1-7.

6. Ashwinkumar V, Krithikadatta J, et al. The effect of reciprocating file motion on microcrack formation in root canals:an SEM study. Int Endod J 2013;47(7):622-627. DOI: 10.1111/iej.12197.

7. Kumaran P, Sivapriya E, et al. Dentinal defects before and after rotary root canal instrumentation with three different obturation techniques and two obturating materials. J Conserv Dent 2013;16(6):522-526. DOI: 10.4103/0972-0707.120968.

8. Didato A, Eid AA, et al. Time-based lateral hygroscopic expansion of a water expandable obturation point. J Dent 2013;41:796-801. DOI: 10.1016/j.jdent.2013.06.012.

9. Highgate DJ, Lloyd JA. Expandable/contractable composition for surgical or dental use. US Pat, 7,210,935, 2007.

10. Prakash R, Gopikrishna V, et al. Gutta-percha - an untold story. Endodontology 2005;2:32-36. 